\title{
Emotion et intérêt dans les études littéraires
}

\section{Vincent Jouve}

\section{(2) OpenEdition}

Journals

Édition électronique

URL : http://journals.openedition.org/edl/606

DOI : $10.4000 /$ edl. 606

ISSN : 2296-5084

\section{Éditeur}

Université de Lausanne

\section{Édition imprimée}

Date de publication : 15 mars 2014

Pagination : 33-46

ISBN : 978-2-940331-34-5

ISSN : 0014-2026

\section{Référence électronique}

Vincent Jouve, «Emotion et intérêt dans les études littéraires », Études de lettres [En ligne], 1 | 2014 mis en ligne le 15 mars 2017, consulté le 18 décembre 2020. URL : http://journals.openedition.org/ edl/606 ; DOI : https://doi.org/10.4000/edl.606 


\section{EMOTION ET INTÉRÊT \\ DANS LES ÉTUDES LITTÉRAIRES}

Partant du principe que l'analyse d'un texte est une forme de lecture élaborée, on examinera les deux moteurs du plaisir narratif - l'intérêt et l'émotion - pour voir ce qu'ils deviennent dans le cadre d'un enseignement: persistent-ils? sous quelles formes? et pour quels bénéfices?

La question posée par la journée d'étude dont est tiré ce volume Comment repassionner l'enseignement de la littérature? - appelle plusieurs remarques. Son présupposé - les études littéraires sont dépassionnées, elles ne captivent plus et se heurtent à l'indifférence - ne va pas de soi. Le problème actuel des études de lettres tient peut-être moins à leur force d'attraction qu'à leur légitimité: en vue de quoi et pour quels résultats enseigne-t-on des textes au référent problématique, dont la plupart sont vieux de plusieurs siècles et qui posent de nombreuses difficultés de lecture? La deuxième question est celle de l'objet: la littérature ne se limite ni au récit, ni à la fiction, et suppose toujours un travail sur les mots: la prise en compte du langage est donc indispensable. Enfin, il semble nécessaire de faire une distinction entre le côté passionnant de la littérature et le côté passionnant de l'enseignement: un texte séduisant n'implique pas forcément un cours inoubliable; et l'inverse est tout aussi vrai. Pourquoi les textes séduisent-ils les lecteurs et pourquoi les cours intéressent-ils les étudiants sont deux questions sensiblement différentes, qui n'appellent pas les mêmes réponses. L’enjeu de cet article sera de comprendre pourquoi. Par commodité, on se limitera au cas du récit.

Avant d'aborder la question de l'enseignement, demandons-nous ce qui rend les récits passionnants. 


\section{Les sources de la séduction narrative}

Il est habituel de faire dépendre l'intérêt narratif de l'émotion, voire de les assimiler: si un récit nous retient, c'est parce qu'il réussit à nous toucher. Intérêt et émotion ne reposent cependant pas sur les mêmes mécanismes et peuvent exister indépendamment l'un de l'autre.

\subsection{Les stimuli de l'intérêt: inattendu et complexité}

A en croire les théories pragmatistes du récit, l'intérêt narratif est lié à l'incertitude: rien de plus ennuyeux qu'un texte entièrement prévisible ${ }^{1}$. Une telle approche se fonde implicitement sur la théorie de l'information de Shannon ${ }^{2}$ : plus une information est inattendue, plus elle est intéressante. "Il a neigé sur Paris au mois d'août» est ainsi un énoncé plus riche que: «Il a neigé sur Paris en décembre».

L'analyse de l'intérêt en termes d'inattendu comprend cependant de nombreuses faiblesses.

En premier lieu, si elle permet d'évaluer l'intérêt de la dynamique narrative une fois la situation installée, elle ne rend pas compte de l'intérêt de la situation ou des objets mis en scène. A la lecture des Trois Mousquetaires, on peut certes se demander si D'Artagnan et ses amis vont récupérer les ferrets de la reine; mais quel est l'intérêt de nous parler des mousquetaires du roi sous le règne de Louis XIII? Il en va de même concernant les objets (pourquoi décrire la casquette de Charles Bovary? le bouclier d'Achille? un quartier de tomate?) et les personnages (à quoi bon nous parler d'Hamlet? de Don Quichotte? de Bardamu?).

En second lieu, une telle approche ne prend pas bien en compte la notion d'«incongru». Or, l'incongru (non pas ce qui est difficile à

I. C'est, aux yeux de Eco, un critère permettant de hiérarchiser les lectures: «Pour ce qui est de la comparaison avec les échecs, un texte narratif peut ressembler aussi bien à un manuel pour enfants qu'à un manuel pour joueurs experts. Dans le premier cas, on proposera des situations de parties assez évidentes (selon l'encyclopédie des échecs), afin que l'enfant ait la satisfaction d'avancer des prévisions couronnées de succès; dans le second cas, on présentera des situations de parties où le vainqueur a hasardé un coup totalement inédit qu'aucun scénario n'avait encore enregistré, un coup tel qu'il passera à la postérité pour sa hardiesse et sa nouveauté, de sorte que le lecteur éprouve le plaisir de se voir contredit" (U. Eco, Lector in fabula, p. 153).

2. Voir C. E. Shannon, W. Weaver, The Mathematical Theory of Communication. 
prévoir, mais ce qui ne convient pas) est un puissant facteur d'accroche narrative. C'est sur l'incongru que jouent nombre de textes humoristiques, parodiques ou fantastiques. L'intérêt de La Métamorphose de Kafka, par exemple, ne se laisse pas formuler en termes de probabilité. Les chances qu'un individu se transforme en cafard ne figurent sur aucune courbe; la valeur du récit est ailleurs: dans ce qu'exprime cette allégorie sur le plan symbolique.

Enfin, la corrélation entre l'intérêt et l'inattendu présuppose la connaissance explicite de l'ensemble des alternatives, ce qui est la faiblesse de toutes les modélisations de la lecture fondées sur la notion de "prévision». Quand un personnage est pris dans un incendie, on peut se demander si oui ou non il va s'en sortir; mais, lorsqu'un personnage s'accoude à la fenêtre pour rêver, l'objet de sa rêverie est potentiellement infini (ce qui n'empêche pas qu'on puisse s'y intéresser).

L'intéressant ne se confond donc pas systématiquement avec l'inattendu. D'où l'intérêt de compléter le modèle de Shannon par celui de Kolmogorov ${ }^{3}$. Au lieu de partir de l'idée que l'intéressant, c'est l'imprévisible, Kolmogorov avance l'hypothèse que l'intéressant, c'est le complexe. Pour la théorie algorithmique de l'information, un objet est d'autant plus complexe qu'il est difficile à "compresser" (au sens informatique du terme). Autrement dit, l'objet le plus complexe est celui qu'on ne peut décrire plus brièvement qu'en faisant une liste exhaustive de ses propriétés: il n'existe pas de règle permettant d'en résumer telle ou telle dimension. Kolmogorov en est arrivé à cette conclusion en tentant de répondre à la question suivante: à quoi voit-on qu'une suite de nombres est tirée au hasard? Une suite tirée au hasard est une suite qui n'obéit à aucune loi, qui n'a été générée par aucun algorithme ${ }^{4}$.

Le texte littéraire, n'étant pas dû au hasard, obéit bien sûr à des algorithmes. L'ambition de la poétique ou de la sémantique structurale est précisément de les identifier en mettant au jour une "grammaire du récit» ou un modèle achronique de la signification. Mais ce qui est intéressant en lui, c'est ce qui échappe (encore) à la modélisation par

3. Pour une présentation accessible de la pensée de Kolmogorov, on pourra se reporter à trois articles de l'Encyclopedia Universalis (signés ou co-signés par J.-P. Delahaye) : "Complexité mathématique», "Complexité algorithmique», "Théorie algorithmique de l'information".

4. De manière schématique, on peut définir l'algorithme comme une recette, une suite finie d'instructions permettant d'aboutir à un résultat. 
algorithmes. Un texte est donc formellement d'autant plus complexe qu'il échappe aux schémas narratifs et sémantiquement d'autant plus complexe qu'il échappe aux schémas interprétatifs. Rappelons cependant qu'aucun texte ne vise la complexité absolue: il serait alors illisible. Toute la difficulté est de trouver un équilibre entre le maintien d'un minimum de repères (pour assurer la lisibilité) et une part de complexité (pour accrocher l'intérêt).

Pour résumer, lorsque je lis un récit, je tente de comprendre ce qu'on me raconte; et, quand je ne dispose pas d'un modèle standard me permettant de comprendre une situation (soit que ce modèle n'existe pas, soit que je l'ignore, soit que j'hésite entre plusieurs modèles), je suis confronté à la complexité. On se demandera, par exemple, pourquoi le vice-consul de Duras tire sur les lépreux dans les jardins de Shalimar ou pourquoi Swann s'entiche d'une femme qui n'est pas son genre.

La tension narrative ne repose donc pas uniquement sur la dialectique du certain et de l'incertain; elle naît aussi de l'écart entre le fait brut et la signification. L'alternative binaire succès/échec ne se pose en effet que pour les genres surcodés comme le récit policier. Dans les grands textes, on ne sait pas ce qui va se passer: la quête des personnages est loin d'être explicite et l'enjeu de la lecture est de la reconstituer. Le lecteur ne se demande pas: voici la quête des personnages, vont-ils la mener à bien?; mais: voici ce que font les personnages; quelle est la quête sous-jacente? Autrement dit, que nous raconte le texte? C'est cela la tension interprétative: non pas l'incertitude sur l'issue, mais l'incertitude sur le sens. Le lecteur ne répond pas à sa façon aux questions posées par le texte; mais répond à sa façon aux questions qu'il a lui-même construites en lisant le texte.

La complexité seule ne suffit cependant pas à susciter l'intérêt. Si je raconte que j'ai croisé un pope dont la croix comportait sept pointes, je m'entendrai répondre par la plupart des gens: «et alors?». Pour qu'une telle remarque suscite l'intérêt, il faut savoir que la croix orthodoxe comprend normalement huit pointes: l'intéressant, ce n'est donc pas seulement ce qui sort de l'ordinaire, c'est ce que l'on remarque, ce qui frappe l'attention. Mais qu'est-ce qui fait qu'un événement retient l'attention? Un moyen indirect de répondre à cette question consiste à observer les paramètres qui influent sur la mémorisation. D'après les recherches 
en psychologie cognitive, on peut retenir trois facteurs principaux: la discrimination, la localisation, la structuration ${ }^{5}$.

Les individus mémorisent d'autant plus facilement une situation qu'elle est facile à discriminer, autrement dit qu'elle tranche avec leurs habitudes. Si je croise un pigeon dans la rue, je ne vais sans doute pas le remarquer; il va se fondre dans le décor ambiant. Mais il n'en ira pas de même si le pigeon en question est rose avec des pois verts ou pèse vingt kilos. On peut penser que si les cas de déviance (les meurtres, les actes barbares, ou plus communément les actes illicites) offrent une excellente accroche narrative, c'est (outre leur forte charge dramatique) en raison de la simplicité de leur discrimination (ils retiennent immédiatement l'attention). On peut en dire autant des personnages atypiques: le monstre sensible (Quasimodo), le bagnard généreux (Jean Valjean), l'enfant au visage mutilé (Gwynplaine dans L'Homme qui rit).

D’une manière générale, les événements ou les personnages sont d'autant plus accessibles qu'ils sont simples à situer. Les repères facilitent la perception, surtout s'ils sont déjà connus du lecteur ou de l'auditeur avant le récit. C'est pourquoi les romans contemporains sont majoritairement réalistes et ancrés dans le présent des lecteurs (même lorsqu'il s'agit de science-fiction). Les récits de vampires ou de zombies se passent en général dans notre monde. En termes de lisibilité, le bénéfice est double: les créatures monstrueuses entraînent une discrimination maximale; le cadre familier facilite l'immersion.

Rappelons enfin qu'en vertu du modèle de Kolmogorov, la complexité d'une structure diminue en présence de symétries, de duplications et plus généralement de formes simples. De fait, plus un récit est structuré, plus il est facile d'accès (il suffit pour s'en convaincre de comparer un film d'Hitchcock à un film de Godard).

\subsection{Les stimuli de l'émotion}

Il est très difficile de savoir d'où viennent les émotions (et je ne me risquerai pas ici à proposer une théorie). En revanche, on peut mettre en évidence les facteurs qui pèsent sur l'intensité émotionnelle. Selon

5. Je me fonde ici - très librement - sur les travaux de J.-L. Dessalles, qui tente de modéliser l'intérêt narratif dans le contexte de l'interaction conversationnelle. Voir, en particulier, "Complexité cognitive appliquée à la modélisation de l'intérêt narratif». 
les approches cognitivistes, l'intensité émotionnelle varie en fonction des facteurs suivants: la proximité, l'improbabilité, la gradualité ${ }^{6}$.

Un événement nous touchera d'autant plus qu'il implique des gens qui nous sont familiers (autrement dit, affectivement proches). La disparition d'un inconnu m'affectera moins que celle d'un parent ou ami, voire d'une simple connaissance. De manière analogue, l'intensité émotionnelle augmentera avec la proximité spatiale (un cambriolage retiendra d'autant plus mon attention qu'il s'est passé dans mon quartier, dans ma rue, en face de chez moi), ou encore avec la proximité temporelle (à niveaux équivalents, une catastrophe récente me bouleversera toujours plus qu'une catastrophe ancienne). On retiendra aussi la proximité sociale: le suicide d'un employé de France Télécom me touchera sans doute plus si je travaille moi-même à France Télécom. Ce mécanisme de proximité fonctionne également dans le récit de fiction où il joue un rôle fondamental dans le système de sympathie: plus on en sait sur un personnage, plus il nous est familier, plus on est ému par ce qui lui arrive 7 .

En second lieu, une situation dramatique l'est d'autant plus qu'elle avait toutes les chances de ne pas se produire. Si l'improbable augmente l'intensité émotionnelle, c'est sans doute qu'il renforce le sentiment d'injustice. L'information «un enfant a sauté sur une mine alors qu'il allait à l'école» est ressentie comme plus révoltante que l'information "un soldat a sauté sur une mine alors qu'il montait à l'assaut». Symétriquement, le lien entre improbable et émouvant explique pourquoi nous devenons insensibles aux drames répétitifs. C'est avec une certaine indifférence que la plupart des gens apprennent, chaque année, que le week-end de la Toussaint a fait des centaines d'accidents. La valeur émotionnelle de l'improbable se retrouve bien sûr dans le récit de fiction. Mais l'improbable ne l'est plus seulement par rapport aux scénarios communs ${ }^{8}$;

6. Voir J.-L. Dessalles, «Le rôle de l'impact émotionnel dans la communication des événements".

7. J'ai longuement étudié ce mécanisme dans L'effet-Personnage dans le roman. Facile à mettre en œuvre, il permet de rendre sympathique à peu près n'importe quel personnage. Ce "coup de force affectif» est régulièrement source de polémiques. La dernière en date concerne le héros-narrateur des Bienveillantes de Jonathan Littell.

8. Les scripts (ou "scénarios communs») sont des suites d'événements qu'on rencontre fréquemment dans la vie quotidienne. Fondés sur l'expérience ordinaire, ils sont largement partagés par les membres d'une culture: "Nous utilisons un savoir spécifique pour interpréter et participer à des événements auxquels nous avons sou- 
il l'est aussi par rapport aux scénarios intertextuels ${ }^{9}$ : un conte qui se terminerait mal, par la mort de la princesse, par exemple, serait ressenti comme particulièrement dramatique.

L'émotion causée par un événement dépend enfin d'un paramètre graduel, comme une plus ou moins grande somme d'argent gagnée ou perdue, un nombre plus ou moins grand de victimes dans une catastrophe, une forme plus ou moins contagieuse d'épidémie. Il semble que, dans toute situation, il existe un seuil au-delà duquel on est dans l'intolérable. On parlera d'«effet de mur» lorsqu'on se rapproche de ce seuil. L'«effet de mur» est un puissant générateur d'émotion. Dans le film Saving Private Ryan de S. Spielberg ${ }^{10}$, l'intensité émotionnelle est directement liée à l'effet de mur: l'enjeu n'est pas tant de préserver une vie que de ne pas franchir une limite insupportable, l'élimination totale d'une fratrie dont trois membres ont déjà été tués:

Ces trois victimes ne sont pas perçues dans le contexte non borné de toutes les victimes possibles de la guerre, mais au sein du réservoir limité de vies qu'une seule famille peut offrir. L'impact émotionnel lié à la proximité de l'absolue limite qui marque l'extermination totale des enfants mâles de cette famille est supposé assez fort pour émouvoir le général Marshall, pourtant parfaitement au fait du nombre total de victimes de la journée ${ }^{11}$.

vent été conviés. Un savoir détaillé spécifique à une situation donnée nous permet de couper court à de longues analyses sur des expériences récurrentes. Nous n'avons pas à nous demander pourquoi le portier veut voir nos billets quand nous allons au théâtre, ou pourquoi l'on doit garder le silence ou même combien de temps on doit rester assis à notre place. Notre savoir sur des situations spécifiques, comme celle d'aller au théâtre, nous permet d'interpréter les propos que les gens tiennent au sujet du théâtre» (R. Schank, R. P. Abelson, cités et traduits par B. Gervais, Récits et actions, p. 164).

9. Contrairement aux scripts, les scénarios intertextuels ne sont pas hérités de l'expérience commune, mais de la connaissance des textes. Lorsqu'il lit des récits appartenant à un même genre, le lecteur s'attend logiquement à retrouver des suites d'actions stéréotypées. D’une façon générale, plus le genre est défini, plus ses lois orientent le déroulement de l'histoire. S'il devient très vite difficile de concevoir une fin heureuse pour Gervaise dans L'Assommoir, c'est dû aux exigences du récit réaliste autant qu'au mouvement dramatique du texte.

Io. Je reprends l'exemple de J.-L. Dessalles, «Le rôle de l'impact émotionnel dans la communication des événements".

II. Ibid., p. 118. 
Dans le récit de fiction, l'intensité de l'émotion est fortement liée à l'effet de mur, qui explique, entre autres, la force d'impact des situations à échéance décisive comme une condamnation à mort (Le Dernier Jour d'un condamné), l'attaque d'un fort (Le Désert des Tartares), l'imminence de la guerre (Un Balcon en forêt) ou la fin du monde (La Guerre des mondes). On notera que ces situations, au fondement du suspense, sont largement exploitées dans les thrillers cinématographiques.

Pour résumer, l'accroche d'un récit repose sur trois facteurs: l'intérêt narratif (que va-t-il se passer?), généré par l'inattendu; l'intérêt herméneutique (qu'est-ce que ça signifie?), généré par la complexité; et la force émotionnelle. Qu'en reste-t-il dans les études littéraires, qui ne sauraient se confondre avec la simple lecture?

\section{Emotion et intérêt dans l'enseignement}

Que devient la séduction narrative dans l'enseignement? et, en particulier, le couple intérêt/émotion?

L'intérêt ne peut plus reposer sur l'inattendu. Les fonctions thymiques du récit - la surprise, le suspense et la curiosité ${ }^{12}$ - ne fonctionnent qu'à la première lecture. Elles reposent sur la dimension temporelle du récit (envisagé comme succession d'événements). L'enseignant, lui, appréhende le texte comme un espace. Profitant d'une vue d'ensemble, il met en parallèle des passages éloignés, explique le début par la fin, commente la partie au regard du tout. D’une manière générale, tout commentaire suppose une première lecture.

Si l'intensité émotionnelle peut se maintenir, c'est qu'elle dépend de paramètres qui ne sont pas tous liés à la narrativité. La proximité, l'improbabilité et la gradualité ne dépendent pas uniquement de la mise en récit. L'étalon de l'improbable, ce n'est pas l'horizon narratif mais

I2. Selon R. Baroni (La Tension narrative), le suspense tient à l'issue incertaine d'une situation connue; la surprise est provoquée par le dévoilement soudain d'une information passée sous silence, et la curiosité est la conséquence d'une information parcellaire. Pour reprendre l'exemple d'Hitchcock (F. Truffaut, Le Cinéma selon Hitchcock, p. 81), on peut distinguer les trois cas suivants: une bombe est cachée sous la table à l'insu des spectateurs - brusquement, elle explose (surprise); on a vu quelqu'un mettre une bombe sous la table et on se demande si elle va exploser (suspense); quelqu'un a mis un objet sous la table sans qu'on sache de quoi il s'agit (curiosité). 
l'encyclopédie de chaque lecteur. Souvent, un événement m'émeut parce qu'il ne correspond pas aux situations auxquelles je m'attends dans la vie. La relecture ne remet pas en cause ce mécanisme. Je peux lire dix fois Les Misérables, je serai toujours touché par la mort de Gavroche (il n'est pas normal qu'un enfant tombe sur des barricades). On pourrait aller jusqu'à dire que la relecture augmente l'intensité émotionnelle. Relisant un récit, on connaît d'autant mieux les personnages qu'on les a fréquentés une première fois ${ }^{13}$ : le critère de proximité joue à plein. Il n'en reste pas moins que l'émotion n'a aucunement besoin de la médiation d'un cours ${ }^{14}$ : le contact direct avec l'histoire suffit largement.

Ne reste donc que l'intérêt herméneutique, qui a tout à gagner d'un regard critique et distancié. Le rôle de l'enseignant est de fournir des outils pour le mettre au jour. Plus précisément, il s'agit de donner des réponses éclairantes et argumentées aux questions que se pose le lecteur quand il est confronté à la complexité. L'intérêt herméneutique peut bien sûr concerner le contenu comme la forme ou le dispositif rhétorique, l'essentiel étant de répondre à la question "qu'est-ce que cela signifie?» ${ }^{15}$.

Dans le cadre d'un enseignement, on aurait donc intérêt à distinguer la valeur narrative (ce récit est-il captivant?) de la valeur herméneutique (que m’apporte ce texte?). Il est en effet habituel de définir les grandes œuvres comme celles qui ajoutent à l'intérêt narratif la gratification d'un capital symbolique. Or si, bien souvent, la valeur narrative s'affadit avec le temps ${ }^{16}$, le capital symbolique, lui, reste intact (et justifie qu'on étudie des œuvres à l'esthétique datée). Quand on s'interroge sur le corpus à étudier, l'intérêt herméneutique est donc un meilleur critère que l'efficacité narrative.

I3. C'est un mécanisme du même ordre qui assure le succès des séries, littéraires ou télévisées. Le retour des personnages d'épisode en épisode crée entre eux et le lecteur (ou le spectateur) une familiarité sans équivalent.

I4. On peut, bien sûr, s'intéresser, comme on l'a fait ici, à ce qui génère l'émotion. Mais on quitte alors le domaine des études littéraires pour celui de l'anthropologie.

15. A titre d'exemples, l'imparfait itératif évoque chez Flaubert l'ennui d'une vie engluée dans la routine et la répétition; l'asyndète renvoie chez Duras au chaos d'une vie intérieure irreprésentable par le discours normé.

I6. Les émotions narratives de base (suspense, surprise, curiosité) reposent sur une encyclopédie qui est largement culturelle. Non seulement, ce qui est surprenant au XVII ${ }^{\mathrm{e}}$ siècle ne l'est plus forcément au XXI ${ }^{\mathrm{e}}$; mais, les scénarios intertextuels ayant tendance à se cristalliser en poncifs, leur efficacité s'en trouve nécessairement affectée. 
La confrontation à la complexité suffit donc largement à passionner les études littéraires ${ }^{17}$. Mais, si on la juge insuffisante, il est possible de recréer didactiquement les autres dimensions de la lecture narrative.

On peut en effet retrouver dans un enseignement trois ressorts fondamentaux du plaisir du récit: l'alternance entre tension et détente; une forme de suspense liée au "comment"; la sensation de "domestiquer l'inattendu" ${ }^{18}$.

A la lecture d'un récit, la tension est fondée sur l'incertitude. C'est le fameux "que va-t-il se passer?» de Barthes ${ }^{19}$. La détente intervient lorsque chaque question a trouvé une réponse - du moins, dans le «texte classique». Dans l'analyse littéraire, la tension n'est plus narrative mais interprétative - elle est créée par le relevé des "dysfonctionnements» ${ }^{20}$, des «lieux d'incertitude» ${ }^{21}$ - et la détente passe par l'application d'une grille de lecture suffisamment convaincante. Ainsi, comme dans le récit, "le déséquilibre dysphorique [...] contient en germe la promesse d'un rééquilibrage euphorique» ${ }^{22}$; et ce rééquilibrage passe, lui aussi, par une harmonie structurale (mais qui, cette fois, est imposée de l'extérieur).

Dans beaucoup de récits, la question du comment est plus intéressante que celle du succès ou de l'échec ${ }^{23}$; car elle ouvre sur un «espace de virtualités» illimité ${ }^{24}$. Il en va de même dans l'enseignement

I7. Qui ne s'est jamais surpris à apprécier une œuvre auparavant mal aimée suite à un éclairage remarquable?

I8. Pour reprendre une formule de J. Bruner, que je cite de seconde main (cf. R. Baroni, La Tension narrative, p. 409).

19. "Narrativement, toute structure dramatique a pour ressort le suspense: comment cela va-t-il finir?" (Nouveaux Essais critiques, p. 166).

20. Le terme est de M. Charles. Il s'agit de ces incohérences - bévues ou accidents concertés - qui sont à la source des diverses interprétations que l'on peut faire d'un texte: "Le dysfonctionnement apparaît comme une condition nécessaire de la dynamique du texte, l'accident qui permet de passer d'un équilibre à un autre» (Introduction à l'étude des textes, p. 167).

2I. On peut les définir, avec J.-L. Dufays, comme "des unités de sens qui suscitent la perplexité de l'ensemble des lecteurs parce qu'elles constituent des infractions aux principes de cohérence et de non-contradiction qui régissent le développement du sens global» (Stéréotype et lecture, p. 156).

22. Voir R. Baroni, La Tension narrative, p. 133.

23. Pensons à la série des Columbo: le meutre étant montré au téléspectateur au tout début de chaque épisode, la question n'est plus «qui est le coupable?», mais «comment le lieutenant va-t-il réussir à le confondre?».

24. Voir R. Baroni, La Tension narrative, p. 278. 
où le "comment étudier?" est souvent plus intéressant que le "quoi étudier?» L'œuvre se présente naturellement comme un terrain de jeu herméneutique ${ }^{25}$. L'explication d'un texte n'est, par définition, jamais achevée. Il est toujours possible d'appliquer à un texte de nouvelles grilles de lecture qui en changeront le sens et la portée. Une telle ouverture n'est cependant possible que dans la mesure où le texte le permet: "Le déplacement incessant d'un texte dans les lectures qu'il subit et l'histoire qu'il traverse, écrit M. Charles, se fait en un lieu repérable, selon des modalités descriptibles. Ce n'est pas n'importe où que s'effectuent ces opérations ${ }^{26}$. Comme le suggère l'auteur d'Introduction à l'analyse des textes, le propre du littéraire est à chercher dans cette capacité à accueillir toujours de nouvelles interprétations.

On rappellera enfin que l'attraction des récits tient au fait que «l'indétermination du futur et du monde s'inscrit dans l'harmonie et l'intelligibilité d'un discours " ${ }^{27}$; le récit, répondant à une logique et pourvu d'un «sens», permet «d'avoir prise sur les tensions qui, dans la vie quotidienne, paraîtraient autrement insupportables " ${ }^{28}$. L'enseignement permet d'obtenir le même effet, y compris avec les textes ouverts et problématiques, qui semblent tourner le dos à toute cohérence: le propre du geste herméneutique est de les inscrire dans un modèle d'intelligibilité ${ }^{29}$. L’enseignant, comme le narrateur (et, à la vérité, bien plus que lui), conforte ainsi l'idée que les choses ne sont pas entièrement contingentes, qu'elles suivent une direction et qu’on a la possibilité de les maîtriser.

Il est courant, quand on veut défendre le plaisir de la lecture, de la penser sur le modèle du jeu ${ }^{30}$. Or, tout jeu ouvre sur deux formes de plaisir, qui se succèdent dans le temps: le plaisir de la découverte et le plaisir

25. Les biblistes, travaillant sur des textes surétudiés depuis des siècles, en ont pleine conscience: la seule possibilité d'en dire encore du neuf est de faire varier les modèles d'analyse.

26. M. Charles, Introduction à l'étude des textes, p. 374.

27. Voir R. Baroni, La Tension narrative, p. 406.

28. Ibid.

29. Ainsi, on peut faire de Finnegans Wake, texte passablement hermétique, une lecture biographique, freudienne, philosophique ou auto-référentielle (liste qui n'est, bien sûr, pas exhaustive).

30. Voir l'essai de M. Picard, La Lecture comme jeu. Mais déjà Eco, dans Lector in fabula, parlait de "rôle du lecteur", de "stratégies", de "succès" et d' "échecs». Barthes, on s'en souvient, définissait le "code herméneutique» comme «l'ensemble des unités qui 
de la maîtrise ${ }^{31}$. Dans une première étape, on se familiarise avec les composants et les propriétés d'un monde alternatif, dont la nouveauté, l'originalité et souvent la beauté sont déjà source de plaisir; dans une deuxième étape, on goûte la satisfaction d'en dominer les mécanismes et de remporter la victoire. Si la première lecture correspond au plaisir de la découverte, on pourrait avancer que la lecture critique (à laquelle nous forme l'enseignant) correspond au plaisir de la maîtrise. Et ce second plaisir n'est pas moins intense que le premier.

Vincent Jouve

Université de Reims

ont pour fonction [...] de formuler une énigme et d'amener son déchiffrement " $(S / Z$, p. 24).

31. C'est particulièrement net dans les jeux vidéo. 


\section{BIBLIOGRAPHIE}

Baroni, Raphaël, La Tension narrative. Suspense, curiosité, surprise, Paris, Seuil, 2007.

Barthes, Roland, S/Z, Paris, Seuil, 1970.

—, Nouveaux Essais critiques, Paris, Seuil, 1972.

Charles, Michel, Introduction à l'étude des textes, Paris, Seuil, 1995.

Delahaye, Jean-Paul, "Complexité mathématique", "Complexité algorithmique», "Théorie algorithmique de l'information", in Encyclopadia Universalis, http://www.universalis-edu.com/encyclopedie/complexite-mathematique/; http://www.universalis-edu. com/encyclopedie/theorie-de-l-information/.

Dessalles, Jean-Louis, "Complexité cognitive appliquée à la modélisation de l'intérêt narratif", http:// www.Dessalles.fr/ papers/Dessalles-06111201.pdf.

—, "Le rôle de l'impact émotionnel dans la communication des événements", in Actes des Quatrièmes Journées Francophones sur les Modèles Formels de l'Interaction, dir. par J. Lang, Y. Lespérance, D. Sadek, N. Maudet, Université Paris-Dauphine, Annales du LAMSADE, 2007, p. 113-125.

Dufays, Jean-Louis, Stéréotype et lecture, Liège, Mardaga, 1994.

Eco, Umberto, Lector in fabula, Paris, Grasset, 1985.

Gervais, Bertrand, Récits et actions, Longueil, Le Préambule, 1990.

Jouve, Vincent, L'effet-Personnage dans le roman, Paris, PUF, 1992.

Picard, Michel, La Lecture comme jeu, Paris, Minuit, 1986.

Shannon, Claude Elwood, Weaver, Warren, The Mathematical Theory of Communication, Champaign, University of Illinois Press, 1949.

Truffaut, François, Le Cinéma selon Hitchcock, Paris, Seghers, 1975. 
